\title{
A Proposed Linear Programming Based Algorithm to Solve Arc Routing Problems
}

\author{
Hashnayne Ahmed* \\ Department of Mathematics, University of Barishal, Barishal-8200, Bangladesh
}

Received: 24 September 2019; Accepted: 15 October 2019; Published: 08 April 2020

\begin{abstract}
A new linear programming-based algorithm has been demonstrated to find the best way for arc routing. In most arc routing problems, the main goal is to minimize the total cost on the lane. To find the minimum cost giving lane, the existing algorithms find the smallest possible sum of weights by ticking the shortest paths [7-12]. The proposed computer-based algorithm is based on focusing the minimized total cost with some constraint criteria of fixed values. The routes are marked with a series of variables that may differ according to the lane choice and more accurately estimates the exact total cost considering the remaining weight. Finally, a stochastic model for a private company vehicle transport has been discussed with some possible solution expectations.
\end{abstract}

Index Terms: Arc Routing Problem, Linear Programming, Proposed Algorithm, Travelling Salesperson Problem, Arc Routing with Uncertainty.

(C) 2020 Published by MECS Publisher. Selection and/or peer review under responsibility of the Research Association of Modern Education and Computer Science

\section{Glossary}

ARP Arc Routing Problem

AR Arc Routing

e-node Eulerian-node

EV Expected Value

VRP Vehicle Routing Problem

WS Wait-and-See

EC Eulerian Circuit

TSP Travelling Salesperson Problem

RP Recourse Policy

CPP Chinese Postman Problem

\footnotetext{
* Corresponding author. Tel.: +8801967341811; ORCID ID: 0000-0002-2136-6816
}

| E-mail address: hashnayneahmed17@gmail.com 


\section{Introduction}

Shortest routes always give the possible minimum cost but not the minimized cost under the given restrictions. The minimized cost will be obtained by considering the possibly changing weight on the lane and cost for that exact weight. The study of finding the total minimized cost has been sectorized into a variety of problems. The vehicle routing problem is recognized as one of the most renowned combinatorial optimization problems as it has many realistic applications in the field of transportation (includes product distribution, collection, logistics, etc.). It is a special kind of arc routing problem and also effective in modeling a variety of optimization cases like routing protocols, network management, stochastic approaches, street mapping, etc. Arc Routing Problems seeks the best lane on its way with the minimized total cost (time or energy). [2,13-17]

Mathematical programs seek to optimize the objective function of decisions. Linear Programming is the mathematical technique for optimizing (maximizing output or minimizing cost) a linear function of several variables. A linear programming problem can be as the following canonical form:

Optimize (Maximize or Minimize) $z=c^{T} x$

Subject to $A x(\geq$, or $=$, or $\leq) b$

and $x \geq 0$

where $x$ is the vector of variables and $c, b$ are co-efficient vectors.

The linear function which has to be optimized is called the objective function and all the conditions expressed by linear relationships are called the constraints of the linear programming problem. [6]

Minimizing the total cost is the perspective of this study with the optimizing concepts as for CPP type problems, the objective function helps to make the decision on the minimized total cost of linear form. The constructed algorithm could be used in every section of arc routing (routing protocols, network management, stochastic approaches, street mapping, VLSI, drone routing, etc.), although in this paper it has been discussed through vehicle routing.

\section{Preliminaries}

\subsection{Eulerian Circuit}

A Eulerian circuit in a graph is a sequence of edges that begins at a vertex of the graph and travels from vertex to vertex by crossing every edge for once and ends at the same vertex. [2]

For example, we may consider the following graph: (The graph has Eulerian circuits named aecdeba and abedcea.)

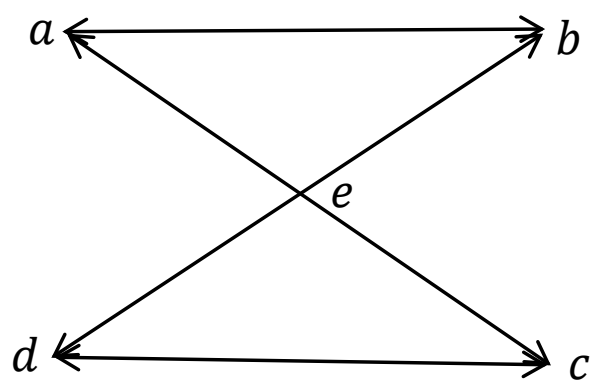

Fig. 1. Eulerian Circuit 


\subsection{Arc Routing}

The process of choosing the best lane on the route is called Arc Routing or Graph Routing. It mainly focuses on the route itself. A graphical problem including an Eulerian circuit will be called a Graph Routing Problem or Arc Routing Problem. [3]

For example, we may consider the famous undirected Chinese Postman Problem. It aims to minimize the total costs such as time or travel distance for the postman. There is another type of problem described by Eiselt [1995] called undirected capacitated arc routing problem. In this case, demands placed on each edge, and each edge must meet the demand.

\subsection{Arc Routing with Uncertainty}

Arc Routing is the study of the best wayfinding for any route which may minimize the total costs like time or travel cost. Arc Routing with uncertainty describes also same, the best wayfinding for any route with the presence of any random parameters on the way. That is, choosing the best way from several possible routes where the objective of routing includes some random parameters where the ultimate focus is minimizing total costs.

If there are $n$ nodes (except depot since it is fixed) on a route and we have to deliver in all nodes with completing an Eulerian circuit (return back to the depot). Then there is total $n$ ! routes that may complete a Eulerian circuit. Although half of the routes are the repeat of another half routes, for the minimized total cost, we have to consider all the $n$ ! possible routes. There is only one way which is the best of these possible routes which will give us the minimized cost (time and travel cost). Now if we consider one of the $n$ nodes have random values like $p$ or $q$, where these two values give us two different ways for minimized cost. This is the consideration of uncertainty in the case of arc routing. Finding the best way in uncertainty situation will be the focus of this study.

For example, we may consider the following graph routing:

Here if we denote the depot with $O$, there are 3 Eulerian nodes $A, B$ and $C$ (nodes for possible Eulerian circuit, shortly, e-node). So, there is $3 !=6$ ways to complete a Eulerian Circuit: $O A B C O, O B C A O, O C A B O, O A C B O, O B A C O, O C B A O$; which will be the facts for arc routing.

Arc Routing is finding the best way from these 6 possible routes. Now if one of the e-nodes $(A, B, C)$ includes some random values, the best possible route finding will be then some subject of concern. In that case, possible routes could be from any of the 6 (not 3), since repeated routes sometimes could give the minimized costs. We may also call it stochastic arc routing. [4] 


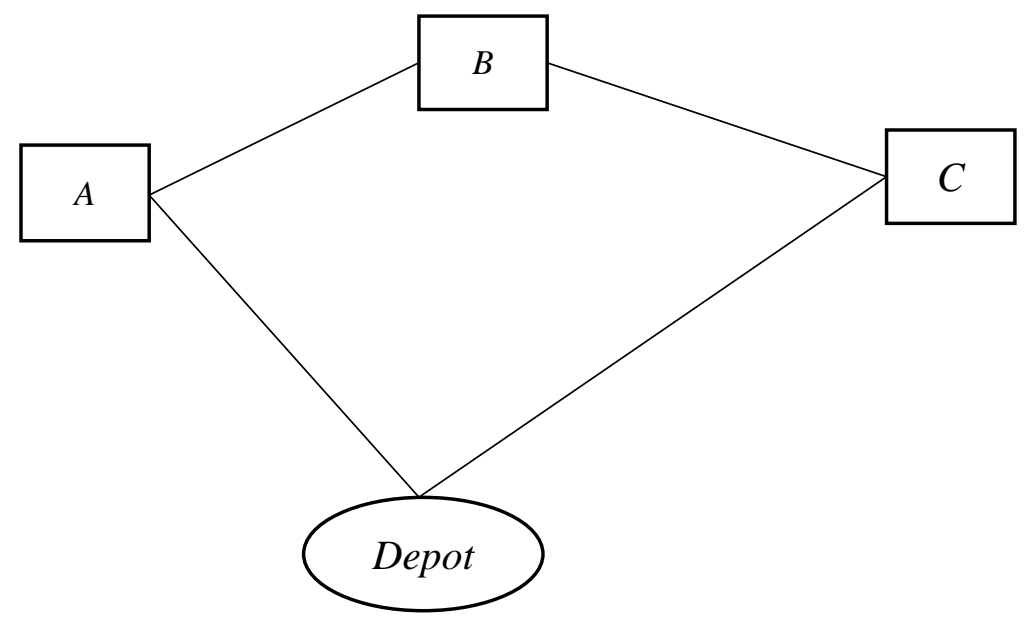

Fig. 2. Arc Routing

\section{Some Observations}

\subsection{Arc Routing with 2 e-nodes}

Let us suppose that $O$ be the depot (same starting and ending point) and $A, B$ are two Eulerian nodes (enodes). There are only two possible ways to complete an Eulerian Circuit (EC): $O A B O$ and $O B A O$. Let $t$ be the total product amount to be delivered and $a, b$ are the required amount product at nodes $A, B$ respectively. Also, let $x_{0}, x_{1}$ and $x_{2}$ be the distances of the first, second and third stages respectively.

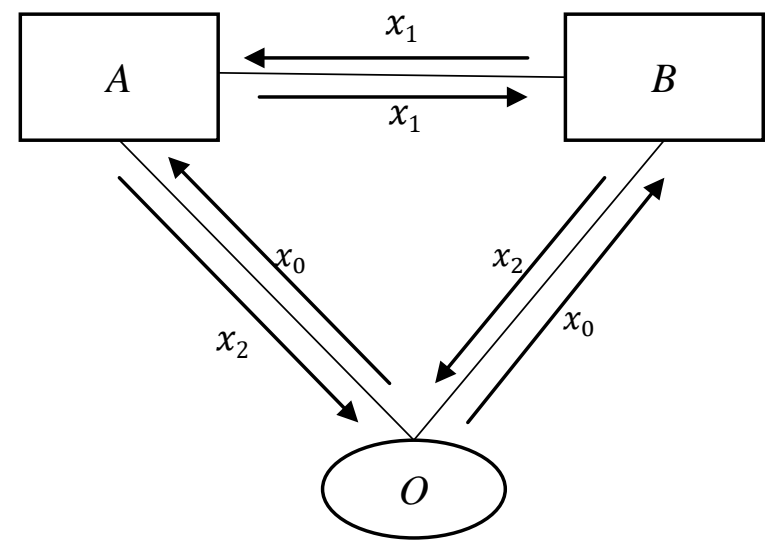

Fig. 3. Arc Routing with 2 e-nodes

Here $t$ is the total amount to be delivered. Some companies always take some more products in the delivery van which may satisfy:

$$
t-a-b \geq 0
$$

Also, the objective is to minimize the total cost. For the above problem, the objective function could be of 
the form:

$\operatorname{Min} x_{0} t+x_{1}(t-a)+x_{2}(t-a-b)$

$=\operatorname{Min} t\left(x_{0}+x_{1}+x_{2}\right)-a\left(x_{1}+x_{2}\right)-b x_{2}$

Example 1. If we take $O A, A B$ and $B O$ as 2,1 and 3 respectively and total deliver capacity for the van is 5 . Then for two e-nodes $(A, B)$ with requirements $a=2$ and $b=2$; there are $2 !=2$ ways: $O A B O$ and $O B A O$.

For $O A B O: x_{0}=2, x_{1}=1, x_{2}=3$ and for $O B A O: x_{0}=3, x_{1}=1, x_{2}=2$

These give the total cost,

$$
\begin{gathered}
T_{C}=\operatorname{Mint}\left(x_{0}+x_{1}+x_{2}\right)-a\left(x_{1}+x_{2}\right)-b x_{2} \\
\text { Subject to, } t-a-b \geq 0 \\
t=5, a=2, b=2 \\
x_{0}=2, x_{1}=1, x_{2}=3 ; \text { or } x_{0}=3, x_{1}=1, x_{2}=2 \\
t, x_{0}, x_{1}, x_{2} \geq 0
\end{gathered}
$$

Solving using LP solver gives $T_{C_{1}}=16$ for $O A B O$ and $T_{C_{2}}=20$ for $O B A O$. That is $O A B O$ is the required path.

\subsection{Arc Routing with 3 e-nodes}

Let us suppose that $O$ be the depot (same starting and ending point) and $A, B, C$ are three Eulerian nodes (enodes). There are $3 !=6$ possible ways to complete an Eulerian Circuit (EC): $O A B C O, O C B A O, O B C A O$, $O A C B O, O C A B O, O B A C O$. Let $t$ be the total product amount to be delivered and $a, b, c$ are the required amount product at nodes $A, B, C$ respectively. Also, let $x_{0}, x_{1}, x_{2}$ and $x_{3}$ be the distances of the first, second, third and fourth stages respectively. We will get these stage distances for every possible EC.

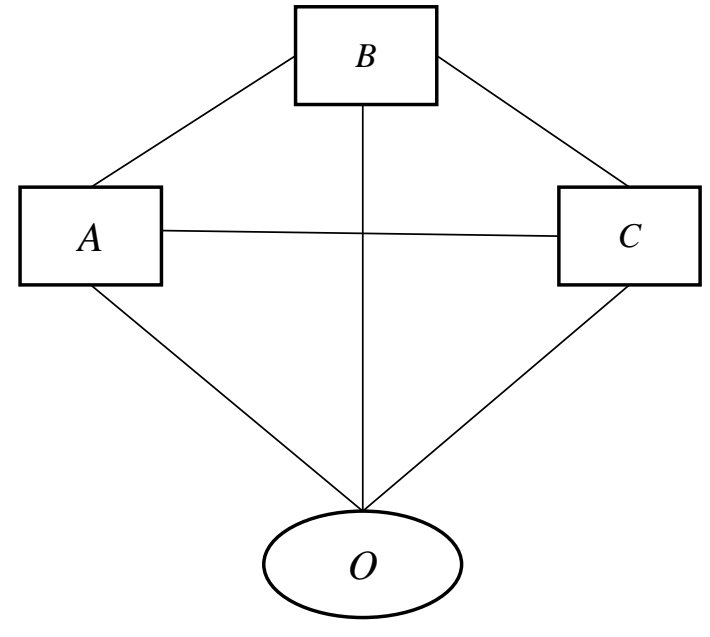

Fig. 4. Arc Routing with 3 e-nodes

Here $t$ is the total amount to be delivered. Some companies always take some more products in the delivery van which may satisfy:

$$
t-a-b-c \geq 0
$$

Also, the objective is to minimize the total cost. For the above problem, the objective function could be of the form: 
Min $x_{0} t+x_{1}(t-a)+x_{2}(t-a-b)+x_{3}(t-a-b-c)$

$=\operatorname{Min} t\left(x_{0}+x_{1}+x_{2}+x_{3}\right)-a\left(x_{1}+x_{2}+x_{3}\right)-b\left(x_{2}+x_{3}\right)-c x_{3}$

\subsection{Arc Routing with n e-nodes}

In a similar manner with $n$ e-nodes with required amounts $\left(a_{1}, a_{2}, \ldots, a_{n}\right)$ and $n+1$ paths to return back to the depot with distances $x_{0}, x_{1}, \ldots, x_{n}$; the objective function could be written as: (total capacity is $t$ )

$$
\begin{gathered}
\operatorname{Min} t\left(x_{0}+x_{1}+\cdots+x_{n-1}+x_{n}\right)-a_{1}\left(x_{1}+\cdots+x_{n}\right)-a_{2}\left(x_{2}+\cdots+x_{n}\right)-\cdots-a_{n-1}\left(x_{n-1}+x_{n}\right)-a_{n} x_{n} \\
\quad=\operatorname{Min} t \sum_{i=0}^{n} x_{i}-a_{1} \sum_{i=1}^{n} x_{i}-a_{2} \sum_{i=2}^{n} x_{i}-\cdots-a_{n-1} \sum_{i=n-1}^{n} x_{i}-a_{n} x_{n}
\end{gathered}
$$

Subject to, $t-a_{1}-a_{2}-\cdots-a_{n} \geq 0$ and all the variables are non-negative.

\section{A proposed Algorithm}

An algorithm has been demonstrated for solving the above arc routing problems by the following steps:

Step 1. Label all the possible $n$ ! routes forming EC with $\mathrm{T}_{i}$, where $i=1,2, \ldots, n, n+1, \ldots, n$ !

Step 2. Take $\mathrm{T}_{i}$ and find all the values of $(i+1)$ stage distances $x_{i}$ for $\mathrm{T}_{i}$. Then solve the linear program

$$
\begin{gathered}
\min T_{C_{i}}=c^{T} x \\
\text { Subject to, } A x=b \\
\sum_{j} a_{j} \leq t ; \quad j=1,2, \ldots, n \\
x, t \geq 0
\end{gathered}
$$

Step 3. Check if $T_{C_{i}}<T_{C_{i+1}}$, then take $T_{C}^{*}=T_{C_{i}}$. Otherwise $T_{C}^{*}=T_{C_{i+1}}$ and return to Step 2. If $T_{C}^{*} \leq T_{C_{i}}$, then stop; it is the minimized total cost.

Remarks. For the algorithm, we may check the example 1 in section 3.1 which estimates the minimized cost.

\section{An Example-Based Scenario Model Concerning Uncertainty}

\subsection{Problem Definition}

Consider a business a company named 'Olympic Industries' in Bangladesh. They sell different packaged food items and some other products in different regions of the country by a central hub in that region from where they deliver their product to different local selling stores and groceries. Their local hub or Depot at Barishal city is located at Bazar Road, from where they deliver their products to different places close to it. For the easiness of the problem formulation, we here consider five places: Natun Bazar, Nathullabadh, Choumatha, Bangla Bazar, and Rupatoli; where they deliver their products in different local stores. We will count the total product amount on these five nodes, and places around these nodes will be included with a nearby one.

Normally, they have some sales representative, who collects data one day before product delivery. They use one motor van for product delivery in these regions. They deliver products twice a week. Here for the scenario, we will consider one delivery with data collected from the company.

For further easier use of these mentioned e-nodes, we will write $O$ for Bazar Road, $A$ for Natun Bazar, $B$ for Nathullabadh, $C$ for Choumatha, $D$ for Bangla Bazar, $E$ for Rupatoli; in the following descriptions of this concept. According to the sales representative in this week, the demand of products (in weight, including no 
return items) in these e-nodes are $100 \mathrm{~kg}$ for $A, 50 \mathrm{~kg}$ for $B, 40 \mathrm{~kg}$ for $D, 60 \mathrm{~kg}$ for $E$, and a random values of $30 \mathrm{~kg}$ or $90 \mathrm{~kg}$ for $C$. Here the product demand in $C$ could be any of the two, depends on some sales store as the sales representative somehow couldn't collect the proper information about them (got shuttered). Note that, the total amount of possible capacity for the delivery van is $300 \mathrm{~kg}$. The company doesn't want to disappoint the selling stores located at node $\mathrm{C}$ since it could permanently damage the sales in those stores.

Following the map, we may conclude the demo figure with the mentioned locations where possible routes are stained.

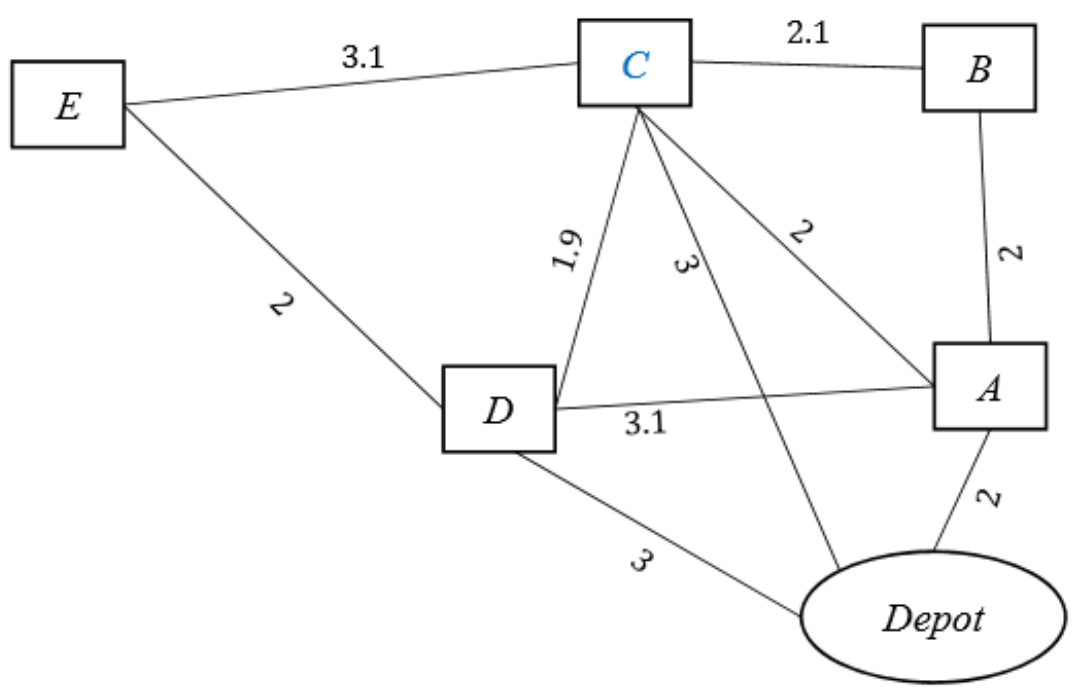

Fig. 5. All Possible Routes with demo figure

Also, the distances for these regions are given in the following distance matrix:

Table 1. Distance Matrix (km)

\begin{tabular}{ccccccc}
\hline & $\boldsymbol{O}$ & $\boldsymbol{A}$ & $\boldsymbol{B}$ & $\boldsymbol{C}$ & $\boldsymbol{D}$ & $\boldsymbol{E}$ \\
$\boldsymbol{O}$ & 0 & 2 & 4 & 3 & 3 & 5 \\
$\boldsymbol{A}$ & 2 & 0 & 2 & 2 & 3.1 & 5.1 \\
$\boldsymbol{B}$ & 4 & 2 & 0 & 2.1 & 5.1 & 5.2 \\
$\boldsymbol{C}$ & 3 & 2 & 2.1 & 0 & 1.9 & 3.1 \\
$\boldsymbol{D}$ & 3 & 3.1 & 5.1 & 1.9 & 0 & 2 \\
$\boldsymbol{E}$ & 5 & 5.1 & 5.2 & 3.1 & 2 & 0 \\
\hline
\end{tabular}

\subsection{Expected solutions}


If the random demand at $C$ is not known in advance, it is discovered after the delivery van reaches $C$. The problem of finding the shortest route to visit all clients starting and ending at the depot is known as the TSP (traveling salesperson problem). In this case, the TSP route is OABCEDO of length $14.2 \mathrm{~km}(2+2+2.1+$ $3.1+2+3$ ). For the expected value solution, we have to forget the uncertainty issues. Then we may observe the following issues: [1]

- When the delivery van arrives at $C$ and demand is 30, it will follow the TSP route. The total demand is $280 \mathrm{~kg}$, which is less than the capacity $300 \mathrm{~kg}$. And so, the traveled distance is $14.2 \mathrm{~km}$.

- When the delivery van arrives at $C$ and demand is 90 , it will follow the route $O A B C E O D O$. The total demand is $340 \mathrm{~kg}$, which is more than the capacity $300 \mathrm{~kg}$. And this results in the traveled distance of $20.2 \mathrm{~km}(2+2+2.1+3.1+5+3+3)$.

- Also, one another route $O C E D O A B O$ could be possible considering uncertainty. It requires a traveled distance of $19.1 \mathrm{~km}(3+3.1+2+3+2+2+4)$.

- If the demand is known before starting the route. The delivery van will follow the TSP route for $30 \mathrm{~kg}$ demand at $C$. Otherwise, it will follow any probable route $O D E C B O A O$ with a traveled distance $18.2 \mathrm{~km}$ $(3+2+3.1+2.1+4+2+2)$.

As first both situations could occur as a possibility of the half, so the expected value is,

$$
E V=\frac{1}{2}(14.2+20.2)=17.2
$$

The probably expected length under optimal recourse policy is,

The wait-and-see solution for known demand is,

$$
R P=\frac{1}{2}(14.2+19.1)=16.65
$$

$$
W S=\frac{1}{2}(14.2+18.2)=16.2
$$

Thus, $W S \leq R P \leq E V$.

Remarks. Some other routes could be possible and it may result in varies of the traveled distance.

\section{Conclusion}

Finding the best way for routing save resources like time, and energy results in minimized cost. In this paper, we presented a linear programming-based algorithm to solve the CPP type arc routing problem for the minimized total cost which ticks the minimized one in every step to find the total minimization. The algorithm doesn't consider the uncertain issues on its way like a traffic jam, speed imitation, weather, delivery priority, etc. For future research, this study will be beneficial in considering the uncertain issues in the algorithm for finding the best lane in all circumstances considered optimization. Stochastic Arc routing is that section to find the best routes considering some uncertain parameters on its way.

\section{Acknowledgements}

The authors would like to thank respectable Shek Ahmed for insightful discussions and offering constructive feedback about this framework. 


\section{References}

[1] Birge, John R., and Francois Louveaux. Introduction to stochastic programming. Springer Science \& Business Media, 2011.

[2] Ahmed, Hashnayne. "Graph Routing Problem Using Euler's Theorem and Its Applications." (2019).

[3] Eiselt, Horst A., Michel Gendreau, and Gilbert Laporte. "Arc routing problems, part II: The rural postman problem." Operations research 43.3 (1995): 399-414.

[4] Toth, Paolo, and Daniele Vigo, eds. Vehicle routing: problems, methods, and applications. Society for Industrial and Applied Mathematics, 2014.

[5] Dror, Moshe, ed. Arc routing: theory, solutions, and applications. Springer Science \& Business Media, 2012.

[6] Ahmed, Hashnayne. "Formulation of Two-Stage Stochastic Programming with Fixed Recourse." Britain International of Exact Sciences (BIoEx) Journal 1.1 (2019): 18-21.

[7] Prim, Robert Clay. "Shortest connection networks and some generalizations." The Bell System Technical Journal 36.6 (1957): 1389-1401.

[8] Kruskal, Joseph B. "On the shortest spanning subtree of a graph and the traveling salesman problem." Proceedings of the American Mathematical Society 7.1 (1956): 48-50.

[9] Dijkstra, Edsger W. "A note on two problems in connexion with graphs." Numerische Mathematik 1.1 (1959): 269-271.

[10] Floyd, Robert W. "Algorithm 97: shortest path." Communications of the ACM 5.6 (1962): 345.

[11] Toth, Paolo, and Daniele Vigo. "An exact algorithm for the vehicle routing problem with backhauls." Transportation Science 31.4 (1997): 372-385.

[12] Guttoski, Pryscila Barvik, Marcos Sfair Sunye, and Fabiano Silva. "Kruskal's algorithm for query tree optimization." 11th International Database Engineering and Applications Symposium (IDEAS 2007). IEEE, 2007.

[13] Assad, Arjang A., and Bruce L. Golden. "Arc routing methods and applications." Handbooks in operations research and management science 8 (1995): 375-483.

[14] Tirkolaee, Erfan Babaee, et al. "An improved ant colony optimization for the multi-trip Capacitated Arc Routing Problem." Computers \& Electrical Engineering 77 (2019): 457-470.

[15] Lacomme, Philippe, Christian Prins, and Wahiba Ramdane-Cherif. "Competitive memetic algorithms for arc routing problems." Annals of Operations Research 131.1-4 (2004): 159-185.

[16] Campbell, James F., et al. "Drone arc routing problems." Networks 72.4 (2018): 543-559.

[17] Poikonen, Stefan, Xingyin Wang, and Bruce Golden. "The vehicle routing problem with drones: Extended models and connections." Networks 70.1 (2017): 34-43. 


\section{Authors' Profiles}

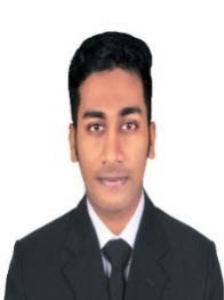

Hashnayne Ahmed (born 7 April 1997) is currently doing his BSc (Hons) degree in Mathematics from the University of Barishal, Bangladesh. His research interests are in Graph Theory, Stochastic Programming, Numerical Analysis, and Fluid Dynamics. He is currently working on the project: A comparative study on Stochastic Programming Problems: modeling and applications in real life. He is a member of the Stochastic Programming Society.

How to cite this paper: Hashnayne Ahmed,"A Proposed Linear Programming Based Algorithm to Solve Arc Routing Problems", International Journal of Mathematical Sciences and Computing(IJMSC), Vol.6, No.2, pp.61-70, 2020. DOI: 10.5815/ijmsc.2020.02.03 